\title{
On Fibonacci and Lucas Vectors and Quaternions
}

\author{
Onur Kaya ${ }^{1, *}$, Mehmet Önder ${ }^{2}$ \\ ${ }^{1}$ Department of Mathematics, Manisa Celal Bayar University, 45140, Manisa, Turkey \\ ${ }^{2}$ Independent Researcher, Delibekirli Village, Tepe Street, 31440, Krkhan, Hatay, Turkey
}

Copyright (C) 2018 by authors, all rights reserved. Authors agree that this article remains permanently open access under the terms of the Creative Commons Attribution License 4.0 International License

\begin{abstract}
In this study, first we investigate the Fibonacci vectors, Lucas vectors and their vector products considering two Fibonacci vectors, two Lucas vectors and one of each vector. We give some theorems for the mentioned vector products and then we give the conditions for such vectors to be perpendicular or parallel. We also introduce the area formulas for the parallelograms constructed by Fibonacci and Lucas vectors with respect to Fibonacci and Lucas numbers. Moreover, we determine some formulas for the cosine and sine functions of the angles between two Fibonacci vectors, two Lucas vectors and lastly a Fibonacci vector and a Lucas vector. Finally, we investigate the Fibonacci quaternions and Lucas quaternions. We give some corollaries regarding the quaternion products of two Fibonacci quaternions, two Lucas quaternions and one of each quaternion. We conclude with the result that the quaternion product of such quaternions is neither a Fibonacci quaternion nor a Lucas quaternion.
\end{abstract}

Keywords Fibonacci Vector, Lucas Vector, Fibonacci Quaternion, Lucas Quaternion

AMS Classsification: 11B39, 11R52, 53A45

\section{Introduction}

Fibonacci numbers are one of the most fascinating subjects of mathematics since they involve many secrets some discovered, some yet to be discovered for over 700 years since they were first introduced. Countless studies were and are being made related to these magnificent numbers. Similar to Fibonacci numbers, another sequence called Lucas numbers were introduced by Edouard Lucas, a French mathematician, in 1878 and they also became a widely studied subject of mathematics [8].

The quaternions were defined by Irish mathematician Sir William Rowan Hamilton [6]. They can be considered as an extension to the complex numbers. During the time that the quaternions were introduced, they had no practical use compared to other methods but recently they are a demanded subject since many algebraic problems on quaternion field have been come across in some applied sciences such as quantum mechanics, analysis, geostatics and differential geometry [10].

Since the Fibonacci numbers became famous, many mathematicians studied different aspects of these numbers and various works were done combining these numbers with algebra, geometry, etc. Fibonacci vectors are one of the most studied subjects along with the Lucas vectors of course [2, 9]. On the other hand, Horadam introduced and generalized Fibonacci quaternions [7]. Akyiğit, Kösal and Tosun studied Fibonacci generalized quaternions and gave some properties [1]. Later, Flaut and Savin studied Fibonacci and Lucas quaternions [3]. Halic1 studied on both Fibonacci quaternions and complex Fibonacci quaternions $[4,5]$.

Considering all the studies mentioned above, in this paper, our goal is to investigate Fibonacci and Lucas vectors and quaternions from a geometrical approach. In the case of vectors, we consider the products of Fibonacci and Lucas vectors. We give some theorems and corollaries regarding the angles between such vectors and areas of parallelograms constructed by Fibonacci and Lucas vectors. Then, we consider the Fibonacci and Lucas quaternions and their quaternion products and we give some important results. 


\section{Preliminaries}

The Fibonacci sequence is the sequence $1,1,2,3,5,8,13,21, \ldots$ where the $n$-th Fibonacci number is denoted with $F_{n}$ for all positive integers $n$ and recurrence relation is given by

$$
F_{n+2}=F_{n+1}+F_{n}, \quad F_{1}=F_{2}=1 .
$$

Fibonacci vectors are defined by

$$
\vec{f}_{n}=\left(F_{n}, F_{n+1}, F_{n+2}\right)
$$

for all integers positive integers $n$ where $F_{i} ; i=n, n+1, n+2$ are Fibonacci numbers [9]. Lucas sequence which is similar to Fibonacci sequence is $1,3,4,7,11,18,29,47, \ldots$ where the $n$-th Lucas number is denoted with $L_{n}$ for all integers $n$ and the recurrence relation is given by

$$
L_{n+2}=L_{n+1}+L_{n}, \quad L_{1}=1, L_{2}=3 .
$$

Lucas vectors are defined by

$$
\vec{l}_{n}=\left(L_{n}, L_{n+1}, L_{n+2}\right)
$$

for all integers $n$ where $L_{i} ; i=n, n+1, n+2$ are Lucas numbers [9].

Theorem 2.1. For all positive integers $n$, let $\vec{f}_{n}=\left[F_{n}, F_{n+1}, F_{n+2}\right]^{t}$. Then, for the matrix

$$
T=\left[\begin{array}{lll}
0 & 1 & 0 \\
0 & 0 & 1 \\
0 & 1 & 1
\end{array}\right]
$$

we have

$$
\vec{f}_{n+1}=T \vec{f}_{n}
$$

The eigenvalues of the matrix $T$ are $\alpha=\frac{1+\sqrt{5}}{2}, \beta=\frac{1-\sqrt{5}}{2}$. Thus, we define the vectors $\vec{a}=\left(1, \alpha, \alpha^{2}\right)$ and $\vec{b}=\left(1, \beta, \beta^{2}\right)$ by using the eigenvalues of the matrix $T$ [9].

Now, we state the following lemmas from [9] that we use in the following sections.

Lemma 2.2. For the numbers $\vec{\alpha}=\left(1, \alpha, \alpha^{2}\right)$ and $\vec{\beta}=\left(1, \beta, \beta^{2}\right)$, we have

$$
\begin{aligned}
& \alpha^{n_{1}} \beta^{n_{2}}+\alpha^{n_{2}} \beta^{n_{1}}=(-1)^{n_{1}} L_{n_{2}-n_{1}}, \\
& \alpha^{n_{1}} \beta^{n_{2}}-\alpha^{n_{2}} \beta^{n_{1}}=(-1)^{n_{1}+1}(\alpha-\beta) F_{n_{2}-n_{1}} .
\end{aligned}
$$

Lemma 2.3. For all positive integers $n$,

$$
\vec{f}_{n}=\frac{1}{\alpha-\beta}\left(\alpha^{n} \vec{a}-\beta^{n} \vec{b}\right)
$$

Lemma 2.4. For all integers $n$,

$$
\vec{l}_{n}=\alpha^{n} \vec{a}+\beta^{n} \vec{b}
$$

Lemma 2.5. For all positive integers $n$,

$$
\left\langle\vec{f}_{n_{1}}, \vec{f}_{n_{2}}\right\rangle=\frac{1}{5}\left(4 L_{n_{1}+n_{2}+2}-(-1)^{n_{1}} L_{n_{2}-n_{1}}\right) .
$$

Lemma 2.6. For all integers $n$,

$$
\left\langle\vec{l}_{n_{1}}, \vec{l}_{n_{2}}\right\rangle=4 L_{n_{1}+n_{2}+2}+(-1)^{n_{1}} L_{n_{2}-n_{1}} .
$$

Lemma 2.7. For all positive integers $n_{1}$ and all integers $n_{2}$,

$$
\left\langle\vec{f}_{n_{1}}, \vec{l}_{n_{2}}\right\rangle=4 F_{n_{1}+n_{2}+2}+(-1)^{n_{1}+1} F_{n_{2}-n_{1}} .
$$

Now, we give a brief summary of quaternions from [6] which we use in the Section 4.

A real quaternion $Q$ is an expression of the form

$$
Q=d+a \vec{i}+b \vec{j}+c \vec{k}
$$

where $a, b, c, d$ are real numbers and $i, j, k$ are the imaginary units and also, they are the unit axis vectors which satisfy

$$
i^{2}=j^{2}=k^{2}=i j k=-1 .
$$


A real quaternion can be given by the form

$$
Q=S_{Q}+V_{Q}
$$

where $S_{Q}=d$ is the scalar part and $V_{Q}=a \vec{i}+b \vec{j}+c \vec{k}$ is the vector part of $Q$. Let $Q=S_{Q}+V_{Q}$ and $R=S_{R}+V_{R}$ be two real quaternions. Then the quaternion product of $Q$ and $R$ is given by

$$
Q \times R=S_{Q} S_{R}-\left\langle V_{Q}, V_{R}\right\rangle+S_{Q} V_{R}+S_{R} V_{Q}+V_{Q} \times V_{R}
$$

The conjugate of $Q=S_{Q}+V_{Q}$ is defined by

$$
\bar{Q}=S_{Q}-V_{Q}
$$

The norm of $Q$ is

$$
N(Q)=\sqrt{Q \times \bar{Q}}=\sqrt{\bar{Q} \times Q}=\sqrt{a^{2}+b^{2}+c^{2}+d^{2}} .
$$

If $N(Q)=1$, then $Q$ is called a unit quaternion [6].

\section{Fibonacci and Lucas Vectors}

In this section, we investigate the vector product of Fibonacci and Lucas vectors. We calculate the vector products and give the results with respect to Fibonacci and Lucas numbers. We also give the angles between Fibonacci and Lucas vectors.

Theorem 3.1. Let $\vec{f}_{n_{1}}$ and $\vec{f}_{n_{2}}$ be two Fibonacci vectors as defined in (1). Then, their vector product is given by

$$
\vec{f}_{n_{1}} \times \vec{f}_{n_{2}}=-(-1)^{n_{1}+1} F_{n_{2}-n_{1}}(1,1,-1)
$$

where $F_{n_{2}-n_{1}}$ is a Fibonacci number.

Proof. From Lemma 2.3, for the Fibonacci vectors $\vec{f}_{n_{1}}$ and $\vec{f}_{n_{2}}$, we have

$$
\begin{aligned}
& \vec{f}_{n_{1}}=\frac{1}{\alpha-\beta}\left(\alpha^{n_{1}} \vec{a}-\beta^{n_{1}} \vec{b}\right), \\
& \vec{f}_{n_{2}}=\frac{1}{\alpha-\beta}\left(\alpha^{n_{2}} \vec{a}-\beta^{n_{2}} \vec{b}\right) .
\end{aligned}
$$

Therefore, with a simple calculation, one gets

$$
\begin{aligned}
\vec{f}_{n_{1}} \times \vec{f}_{n_{2}} & =-\frac{1}{(\alpha-\beta)^{2}}\left(\alpha^{n_{1}} \beta^{n_{2}} \vec{a} \times \vec{b}+\alpha^{n_{2}} \beta^{n_{1}} \vec{b} \times \vec{a}\right) \\
& =-\frac{1}{5}\left(\alpha^{n_{1}} \beta^{n_{2}}-\alpha^{n_{2}} \beta^{n_{1}}\right)(\sqrt{5}, \sqrt{5},-\sqrt{5}) \\
& =-\frac{1}{\sqrt{5}}(-1)^{n_{1}+1}(\alpha-\beta) F_{n_{2}-n_{1}}(1,1,-1) \\
& =-(-1)^{n_{1}+1} F_{n_{2}-n_{1}}(1,1,-1) .
\end{aligned}
$$

Theorem 3.2. Let $\vec{l}_{n_{1}}$ and $\vec{l}_{n_{2}}$ be two Lucas vectors as defined in (2). Then, their vector product is given by

$$
\vec{l}_{n_{1}} \times \vec{l}_{n_{2}}=5(-1)^{n_{1}+1} F_{n_{2}-n_{1}}(1,1,-1) .
$$

Proof. From Lemma 2.4, for the Fibonacci vectors $\vec{l}_{n_{1}}$ and $\vec{l}_{n_{2}}$, we have

$$
\begin{aligned}
& \vec{l}_{n_{1}}=\alpha^{n_{1}} \vec{a}+\beta^{n_{1}} \vec{b}, \\
& \vec{l}_{n_{2}}=\alpha^{n_{2}} \vec{a}+\beta^{n_{2}} \vec{b} .
\end{aligned}
$$

Therefore, for the vector product of these vectors, we have

$$
\begin{aligned}
\vec{l}_{n_{1}} \times \vec{l}_{n_{2}} & =\alpha^{n_{1}} \beta^{n_{2}} \vec{a} \times \vec{b}+\alpha^{n_{2}} \beta^{n_{1}} \vec{b} \times \vec{a} \\
& =\left(\alpha^{n_{1}} \beta^{n_{2}}-\alpha^{n_{2}} \beta^{n_{1}}\right) \vec{a} \times \vec{b} \\
& =(-1)^{n_{1}+1}(\alpha-\beta) F_{n_{2}-n_{1}}(\sqrt{5}, \sqrt{5},-\sqrt{5}) \\
& =5(-1)^{n_{1}+1} F_{n_{2}-n_{1}}(1,1,-1) .
\end{aligned}
$$


Theorem 3.3. Let $\vec{f}_{n_{1}}$ and $\vec{l}_{n_{2}}$ be a Fibonacci and a Lucas vector as defined in (1) and (2). Then, their vector product is given by

$$
\vec{f}_{n_{1}} \times \vec{l}_{n_{2}}=(-1)^{n_{1}} L_{n_{2}-n_{1}}(1,1,-1)
$$

where $L_{n_{2}-n_{1}}$ is a Lucas number.

Proof. From Lemma 2.3 and 2.4, we have

$$
\begin{aligned}
\vec{f}_{n_{1}} & =\frac{1}{\alpha-\beta}\left(\alpha^{n_{1}} \vec{a}-\beta^{n_{1}} \vec{b}\right), \\
\vec{l}_{n_{2}} & =\alpha^{n_{2}} \vec{a}+\beta^{n_{2}} \vec{b}
\end{aligned}
$$

respectively. Thus, from their vector product, it follows

$$
\begin{aligned}
\vec{f}_{n_{1}} \times \vec{l}_{n_{2}} & =\frac{1}{\alpha-\beta}\left(\alpha^{n_{1}} \beta^{n_{2}}+\alpha^{n_{2}} \beta^{n_{1}}\right) \vec{a} \times \vec{b} \\
& =\left(\alpha^{n_{1}} \beta^{n_{2}}+\alpha^{n_{2}} \beta^{n_{1}}\right)(1,1,-1) \\
& =(-1)^{n_{1}} L_{n_{2}-n_{1}}(1,1,-1) .
\end{aligned}
$$

From Theorems 3.1-3.3, we have the following corollary:

Corollary 3.4. The followings are equivalent:

(i) All Fibonacci and Lucas vectors are perpendicular to the vector $(1,1,-1)$.

(ii) A Fibonacci or a Lucas vector always lies on a plane with normal vector $(1,1,-1)$.

Let now consider the parallelograms obtained by the aid of Fibonacci and Lucas vectors. A parallelogram constructed by Fibonacci vectors $\vec{f}_{n_{1}}$ and $\vec{f}_{n_{2}}$ is called a Fibonacci parallelogram. Similarly, a parallelogram constructed by Lucas vectors $\vec{l}_{n_{1}}$ and $\vec{l}_{n_{2}}$ is called Lucas parallelogram. A parallelogram constructed by a Fibonacci vector $\vec{f}_{n_{1}}$ and a Lucas vector $\vec{l}_{n_{2}}$ is called Fibonacci-Lucas parallelogram. Since the area of a parallelogram constructed with the vectors $\vec{u}, \vec{v}$ is calculated by $\|\vec{u} \times \vec{v}\|$, Theorems 3.1-3.3 give us the following corollary:

Corollary 3.5. (i) The area of a Fibonacci parallelogram constructed by $\vec{f}_{n_{1}}$ and $\vec{f}_{n_{2}}$ is $A_{\vec{f}_{n_{1}}} \vec{f}_{n_{2}}=\sqrt{3 F_{n_{2}}-n_{1}}$.

(ii) The area of a Lucas parallelogram constructed by $\vec{l}_{n_{1}}$ and $\vec{l}_{n_{2}}$ is $A_{\vec{l}_{n_{1}} \vec{l}_{n_{2}}}=5 \sqrt{3 F_{n_{2}-n_{1}}}$.

(iii) The area of a Fibonacci-Lucas parallelogram constructed by $\vec{f}_{n_{1}}$ and $\vec{l}_{n_{2}}$ is $A_{\vec{f}_{n_{1}} \vec{l}_{n_{2}}}=\sqrt{3 L_{n_{2}-n_{1}}}$.

Theorem 3.6. The cosine and sine functions of the angle $\theta$ which is between two Fibonacci vectors $\vec{f}_{n_{1}}$ and $\vec{f}_{n_{2}}$ are

$$
\begin{aligned}
\cos \theta & =\frac{4 L_{n_{1}+n_{2}+2}-(-1)^{n_{1}} L_{n_{2}-n_{1}}}{\sqrt{\left(4 L_{2 n_{1}+2}-2(-1)^{n_{1}}\right)\left(4 L_{2 n_{2}+2}-2(-1)^{n_{2}}\right)}}, \\
\sin \theta & =\frac{5 \sqrt{3} F_{n_{2}-n_{1}}}{\sqrt{\left(4 L_{2 n_{1}+2}-2(-1)^{n_{1}}\right)\left(4 L_{2 n_{2}+2}-2(-1)^{n_{2}}\right)}} .
\end{aligned}
$$

Proof. From Lemma 2.5, we have

$$
\begin{aligned}
\left\|\vec{f}_{n_{1}}\right\|^{2} & =\frac{1}{5}\left(4 L_{2 n_{1}+2}-2(-1)^{n_{1}}\right), \\
\left\|\vec{f}_{n_{2}}\right\|^{2} & =\frac{1}{5}\left(4 L_{2 n_{2}+2}-2(-1)^{n_{2}}\right) .
\end{aligned}
$$

Since, $\left\langle\vec{f}_{n_{1}}, \vec{f}_{n_{2}}\right\rangle=\left\|\vec{f}_{n_{1}}\right\|\left\|\vec{f}_{n_{2}}\right\| \cos \theta$ where $\theta$ is the angle between the vectors $f_{n_{1}}, f_{n_{2}}$, then, also from Lemma 2.5 we obtain

$$
\cos \theta=\frac{4 L_{n_{1}+n_{2}+2}-(-1)^{n_{1}} L_{n_{2}-n_{1}}}{\sqrt{\left(4 L_{2 n_{1}+2}-2(-1)^{n_{1}}\right)\left(4 L_{2 n_{2}+2}-2(-1)^{n_{2}}\right)}} .
$$

Analogue to that, since $\left\|\vec{f}_{n_{1}} \times \vec{f}_{n_{2}}\right\|=\left\|\vec{f}_{n_{1}}\right\|\left\|\vec{f}_{n_{2}}\right\| \sin \theta$ using Lemma 2.5 again, we get

$$
\sin \theta=\frac{5 \sqrt{3} F_{n_{2}-n_{1}}}{\sqrt{\left(4 L_{2 n_{1}+2}-2(-1)^{n_{1}}\right)\left(4 L_{2 n_{2}+2}-2(-1)^{n_{2}}\right)}} .
$$


Theorem 3.7. The cosine and sine functions of the angle $\varphi$ which is between two Lucas vectors $\vec{l}_{n_{1}}$ and $\vec{l}_{n_{2}}$ are

$$
\begin{aligned}
& \cos \varphi=\frac{4 L_{n_{1}+n_{2}+2}+(-1)^{n_{1}} L_{n_{2}-n_{1}}}{\sqrt{\left(4 L_{2 n_{1}+2}+2(-1)^{n_{1}}\right)\left(4 L_{2 n_{2}+2}+2(-1)^{n_{2}}\right)}}, \\
& \sin \varphi=\frac{5 \sqrt{3} F_{n_{2}-n_{1}}}{\sqrt{\left(4 L_{2 n_{1}+2}+2(-1)^{n_{1}}\right)\left(4 L_{2 n_{2}+2}+2(-1)^{n_{2}}\right)}}
\end{aligned}
$$

Proof. The proof of this theorem is similar to the proof of Theorem 3.6.

Theorem 3.8. The cosine and sine functions of the angle $\phi$ which is between a Fibonacci vector and a Lucas vector $\vec{f}_{n_{1}}$ and $\vec{l}_{n_{2}}$ are

$$
\begin{aligned}
\cos \phi & =\frac{\sqrt{5}\left(4 F_{n_{1}+n_{2}+2}+(-1)^{n_{1}+1} F_{n_{2}-n_{1}}\right)}{\sqrt{\left(4 L_{2 n_{1}+2}-2(-1)^{n_{1}}\right)\left(4 L_{2 n_{2}+2}+2(-1)^{n_{2}}\right)}}, \\
\sin \phi & =\frac{\sqrt{15} L_{n_{2}-n_{1}}}{\sqrt{\left(4 L_{2 n_{1}+2}-2(-1)^{n_{1}}\right)\left(4 L_{2 n_{2}+2}+2(-1)^{n_{2}}\right)}}
\end{aligned}
$$

Proof. The proof of this theorem is similar to the proof of Theorem 3.6.

\section{Fibonacci and Lucas Quaternions}

A Fibonacci quaternion is defined by

$$
Q_{n}=F_{n}+F_{n+1} \vec{i}+F_{n+2} \vec{j}+F_{n+3} \vec{k}
$$

where $F_{n}$ are the $n$-th Fibonacci number [7]. Considering equation (1) it is possible to rewrite (4) as

$$
Q_{n}=F_{n}+\vec{f}_{n+1} \text {. }
$$

Now, let consider two given Fibonacci quaternions such as $Q_{n}=F_{n}+\vec{f}_{n+1}$ and $R_{k}=F_{k}+\vec{f}_{k+1}$. From the quaternion product of these quaternions, we obtain

$$
Q_{n} \times R_{k}=F_{n} F_{k}-\frac{1}{5}\left(4 L_{n+k+4}-(-1)^{n} L_{k-n}\right)+F_{n} \vec{f}_{k+1}+F_{k} \vec{f}_{n+1}-(-1)^{k} F_{k-n}(1,1,-1) .
$$

A unit Fibonacci vector is given by

$$
Q_{n}^{0}=\frac{1}{\sqrt{3 F_{2 n+3}}}\left(F_{n}+F_{n+1} \vec{i}+F_{n+2} \vec{j}+F_{n+3} \vec{k}\right)
$$

or

$$
Q_{n}^{0}=\frac{F_{n}}{\sqrt{3 F_{2 n+3}}}+\frac{\sqrt{F_{n+1}^{2}+F_{n+2}^{2}+F_{n+3}^{2}}}{\sqrt{3 F_{2 n+3}}} \frac{\vec{f}_{n+1}}{\sqrt{F_{n+1}^{2}+F_{n+2}^{2}+F_{n+3}^{2}}}
$$

which leads us to

$$
\cos \theta=\frac{F_{n}}{\sqrt{3 F_{2 n+3}}}
$$

Therefore, we get

$$
\sin \theta=\frac{\sqrt{F_{n+1}^{2}+F_{n+2}^{2}+F_{n+3}^{2}}}{\sqrt{3 F_{2 n+3}}} .
$$

Thus, we obtain

$$
Q_{n}^{0}=\cos \theta+\vec{S} \sin \theta
$$

where the vector

$$
\vec{S}=\frac{\vec{f}_{n+1}}{\sqrt{F_{n+1}^{2}+F_{n+2}^{2}+F_{n+3}^{2}}}
$$

is called the axis of the unit quaternion $Q_{n}^{0}$. Moreover, it is well known that a unit quaternion is a rotation operator allowing a rotation with the axis $\vec{S}$. Then, since every Fibonacci vector $\vec{f}_{n+1}$ is perpendicular to the vector $(1,1,-1)$, we have the following geometrical interpretation: 
Corollary 4.1. A unit Fibonacci quaternion $Q_{n}^{0}$ allows a rotation with angle $\theta$ on the planes whose normals are always perpendicular to the vector $(1,1,-1)$.

On the other hand, for a Fibonacci quaternion, we may write

$$
Q_{n}=\frac{\alpha \alpha^{n}-\underline{\beta} \beta^{n}}{\alpha-\beta}
$$

which is called the Binet form [5] where $\underline{\alpha}=1+\alpha \vec{i}+\alpha^{2} \vec{j}+\alpha^{3} \vec{k}, \underline{\beta}=1+\beta \vec{i}+\beta^{2} \vec{j}+\beta^{3} \vec{k}$. The quaternions $\underline{\alpha}, \underline{\beta}$ can be written as

$$
\underline{\alpha}=S_{\underline{\alpha}}+\vec{V}_{\underline{\alpha}}, \quad \underline{\beta}=S_{\underline{\beta}}+\vec{V}_{\underline{\beta}}
$$

where $S_{\underline{\alpha}}=1, S_{\underline{\beta}}=1, \vec{V}_{\underline{\alpha}}=\alpha \vec{i}+\alpha^{2} \vec{j}+\alpha^{3} \vec{k}, \vec{V}_{\underline{\beta}}=\beta \vec{i}+\beta^{2} \vec{j}+\beta^{3} \vec{k}$. Thus, for the quaternion products of $\underline{\alpha}, \underline{\beta}$, we have

$$
\begin{aligned}
& \underline{\alpha} \times \underline{\beta}=\underline{\alpha}+\underline{\beta}+\vec{V}_{\underline{\alpha}} \times \vec{V}_{\underline{\beta}}, \\
& \underline{\beta} \times \underline{\alpha}=\underline{\alpha}+\underline{\beta}-\vec{V}_{\underline{\alpha}} \times \vec{V}_{\underline{\beta}}, \\
& \underline{\alpha} \times \underline{\alpha}=2 \underline{\alpha}-\left(1+\alpha^{2}+\alpha^{4}+\alpha^{6}\right), \\
& \underline{\beta} \times \underline{\beta}=2 \underline{\beta}-\left(1+\beta^{2}+\beta^{4}+\beta^{6}\right) .
\end{aligned}
$$

Therefore, for the quaternion product of two Fibonacci quaternions, by using the equations (3) and (5), we get

$$
\begin{aligned}
Q_{n} \times Q_{k}= & \frac{1}{(\alpha-\beta)^{2}}\left[\left(2 \alpha^{n+k}-(-1)^{n} L_{k-n}\right) \underline{\alpha}\right. \\
& +\left(2 \beta^{n+k}-(-1)^{n} L_{k-n}\right) \underline{\beta}-\alpha^{n+k} N(\underline{\alpha}) \\
& \left.-\beta^{n+k} N(\underline{\beta})-\sqrt{5} F_{n-k}^{2}(1,1,-1)\right]
\end{aligned}
$$

The equation (6) is the Binet form of the Fibonacci quaternion product. Yet it is not a Fibonacci quaternion since it involves a vector such $(1,1,-1)$ in its vector part. Now, we investigate the necessary conditions for (6) to be a Fibonacci quaternion. The scalar part of the quaternion (6) must be a Fibonacci number, i.e.,

$$
F_{x}=\frac{2 \alpha^{n+k}+2 \beta^{n+k}-2(-1)^{n} L_{k-n}-\alpha^{n+k} N(\underline{\alpha})-\beta^{n+k} N(\underline{\beta})}{(\alpha-\beta)^{2}}
$$

and also $F_{n-k}=0$ must be satisfied. If $F_{n-k}=0$, then $n=k$. In this case, we get $L_{n-k}=L_{0}=2$ and from (6), we obtain

$$
Q_{n} \times Q_{k}=\frac{1}{(\alpha-\beta)^{2}}\left[2\left(\alpha^{2 n}-(-1)^{n}\right) \underline{\alpha}+2\left(\beta^{2 n}-(-1)^{n}\right) \underline{\beta}-\alpha^{2 n} N(\underline{\alpha})-\beta^{2 n} N(\underline{\beta})\right] .
$$

We may rewrite the last equation as

$$
Q_{n} \times Q_{k}=\frac{\frac{2\left(\alpha^{2 n}-(-1)^{n}\right)}{\alpha-\beta} \underline{\alpha}+\frac{2\left(\beta^{2 n}-(-1)^{n}\right)}{\alpha-\beta} \underline{\beta}}{\alpha-\beta}-\frac{\alpha^{2 n} N(\underline{\alpha})+\beta^{2 n} N(\underline{\beta})}{(\alpha-\beta)^{2}}
$$

to make it look like to Binet form. From (7), it can easily be seen that for $Q_{n} \times Q_{k}$ to be a Fibonacci quaternion, $\alpha^{2 n} N(\underline{\alpha})+$ $\beta^{2 n} N(\beta)=0$ must be satisfied. Since $\alpha^{2 n} \geq 0, \beta^{2 n} \geq 0, N(\underline{\alpha}) \geq 0$ and $N(\underline{\beta}) \geq 0$, then $\alpha^{2 n}=\beta^{2 n}=0$ must be satisfied which leads us to a contradiction. Therefore, we have the following corollary:

Corollary 4.2. The quaternion product $Q_{n} \times Q_{k}$ of two Fibonacci quaternions $Q_{n}, Q_{k}$ cannot be a Fibonacci quaternion.

A Lucas quaternion is defined by

$$
K_{n}=L_{n}+L_{n+1} \vec{i}+L_{n+2} \vec{j}+L_{n+3} \vec{k}=L_{n}+\vec{l}_{n+1}=\underline{\alpha} \alpha^{n}+\underline{\beta} \beta^{n} .
$$

Considering two Lucas quaternions $K_{n}$ and $K_{m}$, from their vector product we get

$$
K_{n} \times K_{m}=L_{n} L_{m}-\left\langle\vec{l}_{n+1}, \vec{l}_{m+1}\right\rangle+L_{n} \vec{l}_{m+1}+L_{m} \vec{l}_{n+1}+\vec{l}_{n+1} \times \vec{l}_{m+1}
$$

Using Lemma 2.6 and and Theorem 3.2 we obtain

$$
K_{n} \times K_{m}=L_{n} L_{m}-4 L_{n+m+2}+(-1)^{n} L_{m-n}+L_{n} \vec{l}_{m+1}+L_{m} \vec{l}_{n+1}+(-1)^{n+1} 5 F_{m-n}(1,1,-1) .
$$


For Binet form, we have

$$
\begin{aligned}
K_{n} \times K_{m}= & \left(\underline{\alpha} \alpha^{n}+\underline{\beta} \beta^{n}\right) \times \underline{\alpha} \alpha^{m}+\underline{\beta} \beta^{m} \\
= & \left(2 \alpha^{n+m}+\alpha^{n} \beta^{m}+\alpha^{m} \beta^{n}\right) \underline{\alpha}+\left(2 \beta^{n+m}+\alpha^{n} \beta^{m}+\alpha^{m} \beta^{n}\right) \underline{\beta} \\
& -\left(\alpha^{n+m} N(\underline{\alpha})+\beta^{n+m} N(\underline{\beta})\right)+\left(\alpha^{n} \beta^{m}-\alpha^{m} \beta^{n}\right) \vec{V}_{\underline{\alpha}} \times \vec{V}_{\underline{\beta}} .
\end{aligned}
$$

Since $\alpha^{n} \beta^{m}+\alpha^{m} \beta^{n}=(-1)^{n} L_{m-n}$ and $\alpha^{n} \beta^{m}-\alpha^{m} \beta^{n}=(-1)^{n+1}(\alpha-\beta) F_{m-n}$ from (3), we get

$$
\begin{array}{r}
K_{n} \times K_{m}=\left(2 \alpha^{n+m}+(-1)^{n} L_{m-n}\right) \underline{\alpha}+\left(2 \beta^{n+m}+(-1)^{n} L_{m-n}\right) \underline{\beta} \\
-\left(\alpha^{n+m} N(\underline{\alpha})+\beta^{n+m} N(\underline{\beta})\right)+(-1)^{2 n+3} 5 \sqrt{5}(1,1,-1) .
\end{array}
$$

The quaternion product $K_{n} \times K_{m}$ is not a Lucas quaternion since the vector part of it involves a vector such $(1,1,-1)$. Therefore, we have the following corollary:

Corollary 4.3. The quaternion product $K_{n} \times K_{m}$ of two Lucas quaternions $K_{n}, K_{m}$ cannot be a Lucas quaternion.

On the other hand, considering a unit Lucas quaternion by

$$
K_{n}^{0}=\frac{K_{n}}{N\left(K_{n}\right)} .
$$

Therefore, similar to Corollary 4.1, we have the following corollary for unit Lucas quaternions:

Corollary 4.4. A unit Lucas quaternion $K_{n}^{0}$ allows a rotation with angle $\varphi$ on the planes whose normals are always perpendicular to the vector $(1,1,-1)$.

Now, we consider a Fibonacci quaternion and a Lucas quaternion given by

$$
\begin{gathered}
Q_{n}=F_{n}+F_{n+1} \vec{i}+F_{n+2} \vec{j}+F_{n+3} \vec{k}=F_{n}+\vec{f}_{n+1}=\frac{\alpha \alpha^{n}-\underline{\beta} \beta^{n}}{\alpha-\beta}, \\
K_{n}=L_{n}+L_{n+1} \vec{i}+L_{n+2} \vec{j}+L_{n+3} \vec{k}=L_{n}+\vec{l}_{n+1}=\underline{\alpha} \alpha^{n}+\underline{\beta} \beta^{n} .
\end{gathered}
$$

From the quaternion product of the given quaternions we have

$$
Q_{n} \times K_{m}=F_{n} L_{m}-\left\langle\vec{f}_{n+1}, \vec{l}_{m+1}\right\rangle+F_{n} \vec{l}_{m+1}+L_{m} \vec{f}_{n+1}+\vec{f}_{n+1} \times \vec{l}_{m+1}
$$

and by using Lemma 2.7 and Theorem 3.3 we get

$$
Q_{n} \times K_{m}=F_{n} L_{m}-4 F_{n+m+4}(-1)^{n} L m-n(1,1,-1) .
$$

For Binet form, we obtain

$$
\begin{aligned}
Q_{n} \times K_{m}= & \frac{1}{\alpha-\beta}\left(\underline{\alpha} \alpha^{n}-\underline{\beta} \beta^{n}\right) \times\left(\underline{\alpha} \alpha^{m}+\underline{\beta} \beta^{m}\right) \\
= & \frac{1}{\alpha-\beta}\left[\left(2 \alpha^{n+m}+(-1)^{n+1} \sqrt{5} F_{m-n}\right)\right. \\
& +\left(-2 \beta^{n+m}+(-1)^{n+1} \sqrt{5} F_{m-n}\right) \\
& +\left(-\alpha^{n+m} N(\underline{\alpha})+\beta^{n+m} N(\underline{\beta})\right) \\
& \left.+(-1)^{n+2} \sqrt{5} F_{m-n} L_{m-n}(1,1,-1)\right]
\end{aligned}
$$

The quaternion product given by (8) is neither a Fibonacci quaternion nor a Lucas quaternion in its present form. In order to eliminate the vector $(1,1,-1)$ let us assume that $m=n$. From (8), we get

$$
\begin{aligned}
Q_{n} \times K_{n} & =\frac{1}{\alpha-\beta}\left(2 \alpha^{2 n} \underline{\alpha}-2 \beta^{2 n} \underline{\beta}-\alpha^{2 n} N(\underline{\alpha})+\beta^{2 n} N(\underline{\beta})\right) \\
& =2 \frac{\alpha^{2 n} \underline{\alpha}-\beta^{2 n} \underline{\beta}}{\alpha-\beta}-\frac{\alpha^{2 n} N(\underline{\alpha})-\beta^{2 n} N(\underline{\beta})}{\alpha-\beta} \\
& =2 Q_{2 n}-\frac{\alpha^{2 n} N(\underline{\alpha})-\beta^{2 n} N(\underline{\beta})}{\alpha-\beta}
\end{aligned}
$$


For $Q_{n} \times K_{n}$ to be a Fibonacci quaternion,

$$
\alpha^{2 n} N(\underline{\alpha})-\beta^{2 n} N(\underline{\beta})=0
$$

must be satisfied. Since the equation (9) has no real root, then $Q_{n} \times K_{m}$ is not a Fibonacci or a Lucas quaternion even if $m=n$. We state this result with the following corollary:

Corollary 4.5. The quaternion product $Q_{n} \times K_{m}$ of a Fibonacci Quaternion $Q_{n}$ and a Lucas quaternions $K_{m}$ cannot be a Fibonacci or a Lucas quaternion.

\section{Conclusions}

In this work, the vector products of Fibonacci and Lucas vectors were investigated. Equations for the angles between Fibonacci and Lucas vectors were given. Also, the area formulas for parallelograms constructed by Fibonacci and Lucas vectors were given. Then, Fibonacci and Lucas quaternions and their quaternion products were investigated. Some corollaries considering the quaternion products of Fibonacci and Lucas quaternions were given. It was found that the quaternion products of Fibonacci and Lucas quaternions cannot be Fibonacci or Lucas quaternions.

\section{REFERENCES}

[1] M. Akyiğit, H.H. Kösal, M. Tosun, Fibonacci Generalized Quaternions, Advances in Applied Clifford Algebras, 24, 631641 (2014).

[2] K.T. Atanassov, V. Atanassov, A. G. Shannon and J.C. Turner, New visual perspectives on Fibonacci numbers, World Scientific (2002).

[3] C. Flaut, D. Savin, Quaternion Algebras and Generalized Fibonacci-Lucas Quaternions, Advances in Applied Clifford Algebras, 25, 853-862 (2015).

[4] S. Halıc1, On Complex Fibonacci Quaternions, Advances in Applied Clifford Algebras, 23, 105-112 (2013).

[5] S. Halıc1, On Fibonacci Quaternions, Advances in Applied Clifford Algebras, 22, 321-327 (2012).

[6] W.R. Hamilton, Elements of Quaternions, Longman, Green, \& Company, London, 1866.

[7] A.F. Horadam, Complex Fibonacci Numbers and Fibonacci Quaternions, The American Mathematical Monthly, 70(3), 289-291 (1963).

[8] T. Koshy, Fibonacci and Lucas numbers with applications, A Wiley-Interscience Publication, USA, 2001.

[9] E. Salter, Fibonacci Vectors, University of South California, Master Thesis, 2005.

[10] J.L. Wu, Distribution and estimation for eigenvalues of real quaternion matrices, Computers and Mathematics with Applications, 55, 1998-2004 (2008). 